
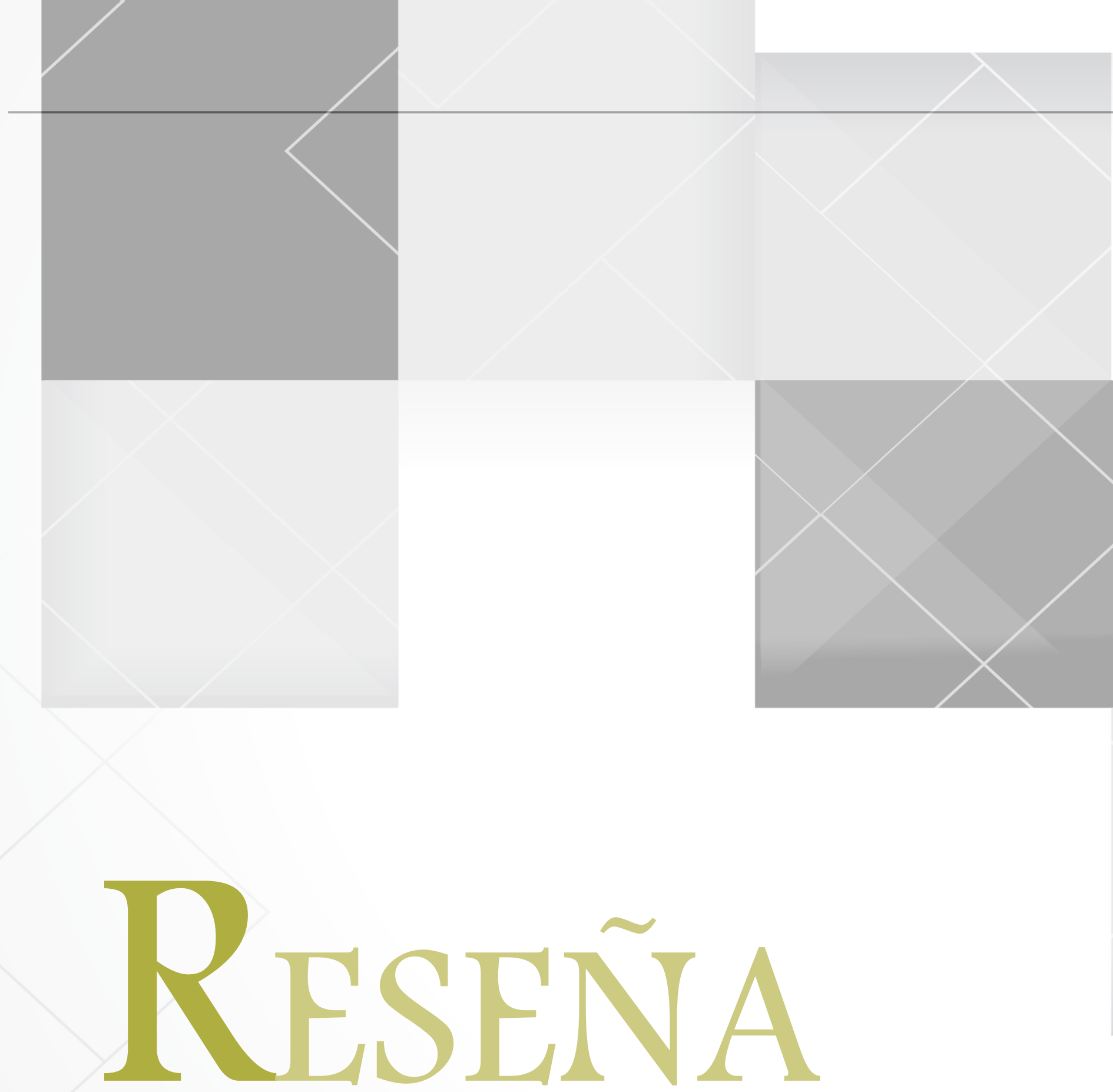

bibliográfica 
$\equiv$

I

1
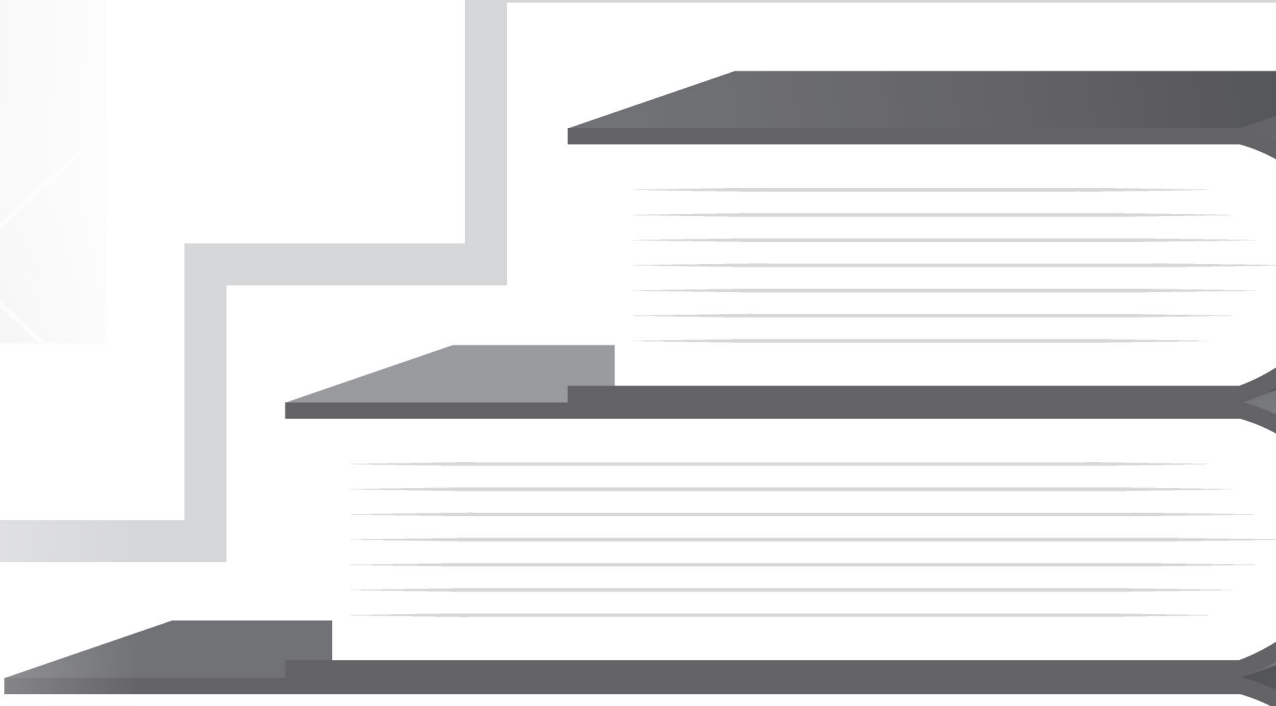


\section{La práctica del liderazgo adlaptativo. Las herramientas y tácticas para cambiar su organización y el mundo}

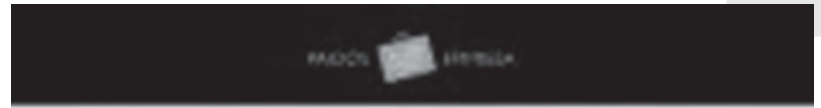

Ronald Heifetz Alexander Grashow Marty Linsky

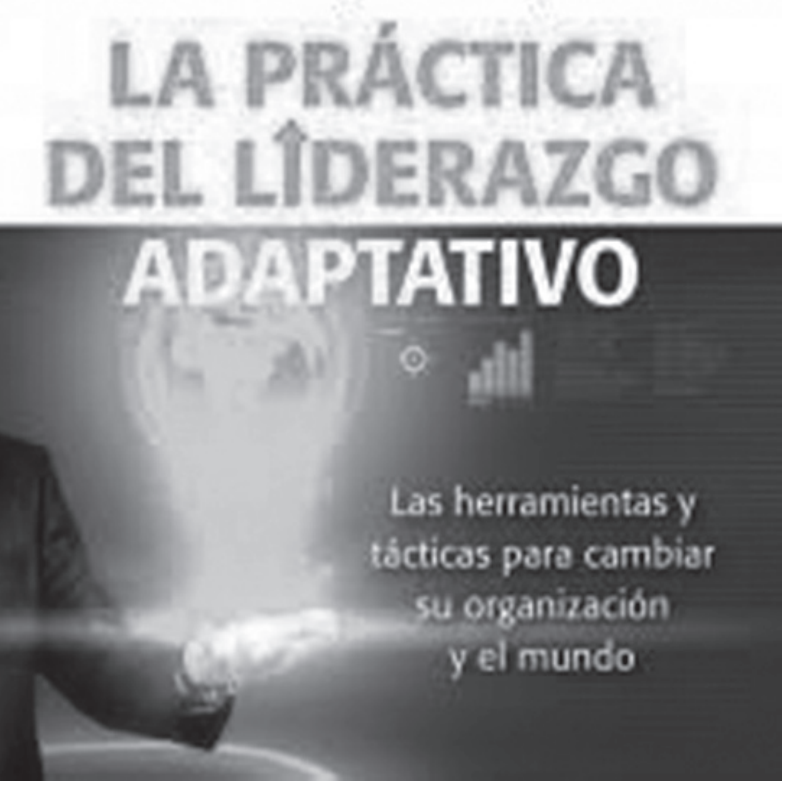

Autores:

Ronald Heifetz, Alexander Grashow Marty Linsky

\section{Editorial: Harvard Business Review Press, 2009}

Reseña elaborada por: Diego Fernando Cardona Madariaga, PhD*

* Ingeniero, especializado en Gerencia; MSc y PhD en Ciencias Administrativas, con Posdoctorado en docencia mediada con TIC. Su desarrollo profesional ha estado enmarcado en la aplicación estratégica de TI como alto directivo gubernamental y consultor con agencias de apoyo multilateral para gobiernos latinoamericanos. De manera paralela ha permanecido vinculado con el sector académico como investigador y colaborador en prestigiosas universidades. En la actualidad se desempeña como vicerrector académico de la Universidad EAN. 
$\mathrm{E}$ n octubre de 2013, tuve la fortuna de asistir en Bucaramanga a una conferencia de dos íconos de la Escuela de Negocios de Harvard; por un lado, Michael Porter, padre de la competitividad; y por otro, Ronald Heifetz, creador del concepto de liderazgo adaptativo. Ahora, en mi función de vicerrector académico de la Universidad EAN y en medio de un periodo de profundos cambios institucionales, han venido a mi memoria las pertinentes recomendaciones de Heifetz, autor del texto «La práctica del liderazgo adaptativo», que con la coincidencia de la visita de dos miembros de nuestra Sala General a Harvard, me puse en la tarea de armar una breve reseña del interesante texto en la expectativa de picar la curiosidad del lector e invitarlo a revisarlo en profundidad para que lo conozca, lo medite y en lo posible, lo aplique.

El proceso de cambio en el que está inmersa la Universidad EAN y al que se encuentran avocadas la gran mayoría de las organizaciones como resultado del turbulento entorno actual, exige un estilo de liderazgo innovador, que en la misma tónica de Goldsmith \& Reiter, indican que el éxito pasado no asegura el éxito futuro, lo que implica revaluar las tradicionales estrategias y desarrollar competencias complementarias en los equipos de trabajo. Esa propuesta la describen Heifetz, Grashow \& Linsky en un texto interactivo y ameno que presenta el marco teórico necesario acompañado de herramientas prácticas que demuestran su pertinencia y eficacia a lo largo de cinco secciones con 23 capítulos.

La sección I del texto, indica, que el propósito de los autores es ofrecer una herramienta de consulta y aplicación, y por lo tanto, es posible leerlo ordenadamente o simplemente hojearlo para encontrar lo que interesa dado que está escrito para ser leído, comprendido y aplicado gracias a la incorporación de preguntas, ejercicios, gráficos y otras herramientas. En esta sección se aborda la teoría requerida que ha sido desarrollada a profundidad en un par de libros previos titulados «Liderazgo sin respuestas fáciles» y «Liderazgo sin límites», en los que se define que el liderazgo adaptativo es la práctica de movilizar a las personas para que afronten desafíos difíciles y prosperen en el sentido de la biología evolutiva en el que la adaptación exitosa tiene tres características: preservar el ADN que garantiza la supervivencia; descartar el ADN innecesario; y crear el ADN necesario para adaptarse a desafiantes entornos combinando aproximaciones tradicionales con nuevas competencias para hacer frente a retos sin precedentes. El liderazgo adaptativo propone cambios que potencian la capacidad de prosperar, se construye aprovechando el pasado sin deshacerse de él e implica experimentación; así mismo, depende de la diversidad y de los valores. Las adaptaciones resultantes tienen el potencial para desplazar, volver a regular y reordenar las antiguas estructuras a lo largo de periodos de tiempo extensos. En el liderazgo adaptativo no se trata de hacer converger las expectativas, se trata de desafiarlas y presionar a los individuos llevándolos al límite. Ahora 
bien, el tipo de liderazgo que se propone busca generar soluciones a desafíos adaptativos y no a problemas técnicos, entendiendo los adaptativos como aquellos cuya definición y solución requiere de aprendizaje, mientras que los técnicos tienen definiciones y soluciones claras. Por otro lado, la adaptación es un proceso de conservación de aquello que es esencial, y al mismo tiempo es un proceso de pérdida de aquello que permite evolucionar en un entorno altamente turbulento que requiere de un líder que no confunda el liderazgo con la autoridad, el poder y la influencia. Los tres últimos son excelentes herramientas que por sí solas no definen el liderazgo. Es necesario mantener a la organización en una tensión continua entre lo antiguo y lo nuevo, entre la zona de confort y la innovación, en la misma línea que proponen Pascale, Millemann y Gioja en su interesante texto «El líder en tiempos de caos».

La propuesta de liderazgo implica tres actividades clave: observar, interpretar y diseñar intervenciones basadas en lo observado e interpretado, teniendo en cuenta que los individuos perciben de manera diferente la realidad dependiendo de las experiencias previas y perspectivas. Observar, es una actividad subjetiva y el liderazgo adaptativo busca que sea lo más objetiva posible. Interpretar, es más complejo porque se corre el riesgo de enfrentar diferencias, sin embargo, es imposible evitar hacer interpretaciones, y por ello, para ejercer el liderazgo adaptativo es necesario dedicar tiempo a la reflexión antes de actuar a pesar de no disponer de toda la información necesaria para hacerse una idea completa y ver la realidad desde puntos de vista diferentes. Para cerrar el ciclo, se debe intervenir la realidad aportando contexto y conectando la interpretación con el propósito para que todos perciban que se trata de un esfuerzo colectivo que tiene en cuenta los recursos y habilidades disponibles en la organización. El texto desarrolla los aspectos teóricos expuestos previamente en cuatro secciones que expresan los dos procesos fundamentales de cualquier práctica profesional, diagnosticar y actuar, las cuales se despliegan en dos dimensiones claves; por un lado, la sistémica y por otro la personal. En cada uno de los capítulos se encontrarán ideas y ejemplos seguidos de «Reflexiones en el palco» y «Trabajo de campo» que hacen del texto una herramienta de aplicación en la realidad de las organizaciones y a nivel personal. Antes de comprometerse con un proceso de cambio es recomendable no hacerlo solo, y por ello, vale la pena involucrar al equipo de trabajo y distribuir responsabilidades, teniendo en cuenta que el mejor laboratorio para aprender de liderazgo adaptativo es la vida misma, evitando en lo posible pasar a la acción sin un proceso previo de reflexión; es deseable tomar decisiones difíciles y disfrutarlas.

La sección II, aborda la necesidad de diagnosticar el sistema y se desarrolla en cuatro capítulos; el primero, describe la misión, fortalezas y debilidades de la organización que justifican la necesidad del cambio. El siguiente capítulo ayuda a identificar los desafíos adaptativos a través de características tales 
como que existen diferencias entre lo deseado y la realidad; las soluciones actuales son inadecuadas y requieren aprendizaje complejo, implica a nuevos participantes, demanda más tiempo y genera una sensación de desequilibrio en la medida que se empieza a percibir la crisis. La clasificación de los desafíos permite llegar a cuatro arquetipos; el primero, corresponde a discrepancias entre la conducta y los valores que se defienden, por ejemplo, afirmar que se cree en la innovación pero se tiene poca tolerancia al fracaso; el segundo, está asociado a compromisos contradictorios, por ejemplo, proponer metas de crecimiento disruptivas pero no entregar los recursos requeridos; el tercero, implica nombrar lo innombrable, por ejemplo, decir en público sólo una parte de lo que realmente se piensa; y el último, es la típica procastinación; por ejemplo, enfocarse sólo en una parte del problema y desviar la responsabilidad. Continua el texto proponiendo el diagnóstico del entorno político de la organización, de las lealtades y alianzas, los valores detrás de las acciones y evalúa la organización, porque comprender las relaciones políticas es clave para ver cómo la organización funciona en un conjunto de partes que se relacionan entre sí. Por ello, es recomendable identificar para cada grupo de interés las posibles objeciones al proceso ¿Cómo se verá afectado por la solución del desafío? Los resultados deseados ¿Qué le gustaría lograr con la solución de la cuestión? El nivel de compromiso ¿Qué tanto se preocupa del tema y de la organización? Y el grado de poder e influencia ¿Qué recursos controla y quién los requiere? Igualmente, es recomendable identificar los valores ¿Cuáles son los compromisos y creencias que guían la toma de decisiones? Las lealtades ¿Qué obligaciones tienen? Los riesgos ¿Qué temen perder con el cambio? Y las alianzas ocultas ¿Qué intereses compartidos tienen? Finaliza esta sección con un capítulo que presenta las características de una organización adaptativa con una herramienta de medición práctica que permite verificar si se pueden mencionar los «elefantes», sin problema, si la responsabilidad por el futuro es compartida, si el equipo de trabajo tiene un juicio independiente de los directivos, si la capacidad de liderazgo está desarrollada y si está institucionalizada la reflexión y el aprendizaje continuo. Se puede concluir que el diagnóstico implica distinguir los desafíos técnicos de los adaptativos, entender los conflictos desde la posición del individuo, ser capaz de leer entre líneas, es decir, entender e interpretar a los individuos, y finalmente, buscar las figuras de autoridad dado que sus conductas permiten intuir el impacto de las acciones que se implementen.

La sección III, que trata de la acción sobre el sistema, propone acciones que los líderes deberían realizar para implementar el cambio, sin embargo, es recomendable no actuar sin consultar al equipo de trabajo para probar el diagnóstico y buscar alternativas que tomen en cuenta efectos no deseados. En un primer capítulo propone migrar de las interpretaciones técnicas propias de los sistemas tradicionales que se basan en datos y hechos y buscan alternativas conocidas, a interpretaciones adaptativas que implican pensar 
sistémicamente y aceptar la incertidumbre y el conflicto. Para lograrlo es recomendable estar atento al equipo de trabajo dado que normalmente tenderán a ser más técnicos que adaptativos, intentar reformular la estructura de las interpretaciones iniciales del equipo de trabajo invitándolos a ser creativos, a proponer múltiples interpretaciones y escuchar las ideas antes que defenderlas. Un segundo capítulo recomienda realizar intervenciones consensuadas, que aseguren el apoyo de los interesados y que con su retroalimentación generen cambios impactantes mediante siete tácticas: suba al balcón, determine la madurez del problema, reconozca su papel en el problema, plantee el reto constructivamente, resista la tentación de retractarse de su intervención, analice las facciones que surjan y mantenga el enfoque. El tercer capítulo de esta sección, describe una situación de la que deben ser conscientes quienes lideren los procesos de cambio y es que existirán aliados y antagonistas, describiendo alternativas de acción para manejarlos. En el siguiente capítulo de esta sección se indica, que dado que seguramente se tendrán que enfrentar conflictos, estos se deben observar como oportunidades, motores para la creatividad y la innovación y no como obstáculos; se cierra retomando la organización y las calidades asociadas a su adaptabilidad de tal manera que el líder del proceso de cambio fomente competencias como el pensamiento independiente y el desarrollo profesional. Para gestionar el conflicto se proponen cuatro alternativas; primero, crear un ambiente de contención en el que se puedan plantear las situaciones conflictivas sin eludirlas. Puede ser un espacio físico donde los individuos se sientan a salvo y ello implica que el líder mantenga la tensión en un nivel productivo sin llevar a la organización al caos. En segunda instancia se requiere controlar la presión, elevándola inicialmente para generar la tensión requerida y luego bajándola a niveles tolerables por la organización. En tercera instancia se ha de regular el esfuerzo emocional y conceptual. El cambio genera un dilema emocional porque implica renuncias que se deben solicitar a la velocidad adecuada; finalmente, el conflicto se puede gestionar mostrando el futuro para recordar la visión positiva que hace que el esfuerzo valga la pena.

La sección IV, que trata del diagnóstico del individuo, profundiza en el lado personal del desarrollo de las prácticas de liderazgo adaptativo. Para comenzar, los autores proponen que los individuos se deben ver como sistemas complejos con múltiples identidades que se adaptan a las situaciones que enfrentan en el devenir en la organización. Por ello, se discute la importancia de las lealtades en el lugar de trabajo, la comunidad y sus raíces culturales, nacionales, étnicas y espirituales. Como en todo el libro, se propone como herramienta de aplicación práctica un conjunto de preguntas reflexivas para reconocer los problemas que generan reacciones y cómo enfrentarlas. Igualmente, invita al lector a explorar sus tolerancias y ampliar sus redes en lo que los autores denomina «ampliar su ancho de banda». Nuevamente, en la 
perspectiva de aplicación práctica, se proponen herramientas para identificar el alcance de la autoridad, verificando las diferencias entre la formal y la informal y el papel del líder en el proceso de cambio de la organización en el que se deben priorizar los propósitos.

La sección $V$, que trata de la acción sobre el individuo, provee instrucciones para continuar expandiendo las prácticas del liderazgo adaptativo logrando el compromiso personal con la organización sin perder identidad. Recomiendan mantenerse conectado a los propósitos personales, siendo conscientes de su evolución y del peligro de generar percepciones negativas en el equipo de trabajo para lo cual es esencial comprometerse a fondo con el proceso, de tal manera que se controlen sentimientos de incompetencia o el miedo natural a cometer errores ante situaciones difíciles. Los capítulos finales del texto apuntan a recomendar acciones para fomentar el carisma requerido por un líder, incluyendo tomar clases de actuación para expresar emociones y asumir riesgos, inspirar al equipo de trabajo identificando propósitos comunes, hablando desde el corazón y expresando sus sentimientos. Es recomendable ser consciente de que todo lo que se hace cuando se lidera un cambio adaptativo es un experimento con lo cual cada acción se puede observar como un intento que no es necesariamente una decisión final, y por ello, es posible asumir riesgos, exceder la autoridad y liberase de la presión. El cierre del texto es disiente, «prosperar», propone acciones para mantener un estilo de liderazgo adaptativo en plazos viables de tiempo gracias a la capacidad de fomentar redes personales, buscar confidentes, ser conscientes de que hay vida fuera de la organización, y tal vez lo más importante, mantener el optimismo.

Para finalizar, es recomendable revisar con ánimo de inventario la propuesta, dado que hay situaciones en las que claramente es útil, pero hay otras en las que posiblemente no debería usarse porque propone acciones como asumir riesgos y crear desequilibrios que podrían afectar a la organización o que no son de fácil aceptación dada su cultura. Por otro lado, es relevante verificar las habilidades de los individuos para usar los conceptos y herramientas descritas en el libro, teniendo en cuenta que a pesar de que son técnicamente correctas, la complejidad de su aplicación implica que son apropiadas para alguien con experiencia en dirección, y tal como lo describen los autores, debe ser alguien con capacidad para deshacerse del espejismo de sistemas obsoletos, distinguir problemas técnicos de desafíos adaptativos, diferenciar liderazgo de autoridad, vivir en la productividad del desequilibrio, observar, interpretar e intervenir, comprometerse y conectarse con un propósito.

En los anteriores términos, y como lo indiqué al inicio, invito a conocer, meditar, y, en lo posible, aplicar la propuesta de liderazgo adaptativo en la búsqueda del éxito de los procesos de cambio en los que se encuentran sus organizaciones. 

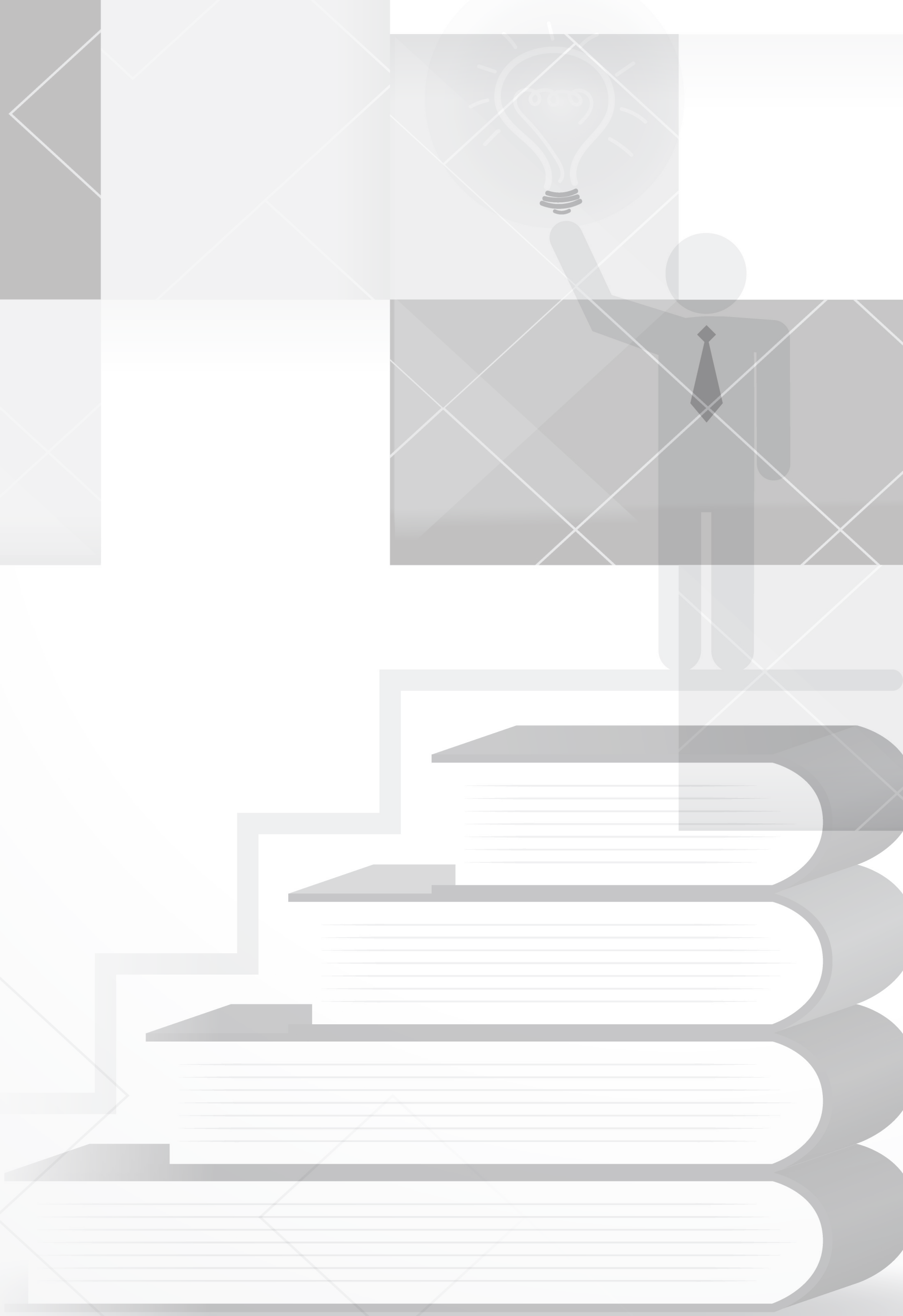